Preface

\title{
Maintaining Hemostasis and Preventing Thrombosis in Coronavirus Disease 2019 (COVID-19)-Part III
}

\author{
Emmanuel J. Favaloro, PhD, FFSc (RCPA) ${ }^{1}$ Giuseppe Lippi, MD² \\ ${ }^{1}$ Department of Haematology, Sydney Centres for Thrombosis and \\ Haemostasis, Institute of Clinical Pathology and Medical Research \\ (ICPMR), Westmead Hospital, Westmead, Australia \\ 2 Section of Clinical Biochemistry, University of Verona, Verona, Italy \\ Semin Thromb Hemost 2022;48:3-7.
}

Welcome to another issue of Seminars in Thrombosis and Hemostasis (STH). This issue is published under the "continuing banner" of "Maintaining hemostasis and preventing thrombosis in coronavirus disease 2019 (COVID-19)," this being Part III, or the third such issue. The first two issues were respectively published in $2020^{1}$ and $2021^{2}$ and proved very popular with the STH readership. COVID-19 is caused by severe acute respiratory syndrome coronavirus 2 (SARSCoV-2). Believed to originate from Wuhan City in China, with the first reported case in December 2019, at time of writing, there were over 200 million cases reported worldwide, and over 4.3 million attributable deaths. ${ }^{3}$

This compares with 113 million cases reported worldwide, and over 2.5 million attributable deaths, at the time of writing the second issue's Preface (February 27 ${ }^{\text {th }}, 2021$ ), ${ }^{2}$ and 26 million cases reported worldwide, and nearly 900,000 attributable deaths, at the time of writing the first COVID-19 issue Preface (August 30th, 2020). ${ }^{1}$ From a smattering of reports in the scientific literature in late $2019(n=228)$, there are now over 165,000 publications ascribed to COVID-19 in PubMed, including over 18,000 reviews. This compares to 100,000 publications, including almost 12,000 reviews, at time of writing the second COVID-19 issue Preface, ${ }^{2}$ and 51,000 publications, including over 5000 reviews, at time of writing the first COVID-19 issue Preface. ${ }^{1}$ Thus, cases of COVID-19 have grown fourfold, and attributable deaths over threefold, since writing the first Preface. We are hopeful that this trend that has now finally start to slow down in several countries, assisted by the development and use of several vaccines against severe acute respiratory syndrome coronavirus 2 (SARS-CoV-2). ${ }^{4-6}$ Publications have kept pace, and those related to COVID-19 have tripled since the first issue Preface, just over 1 year ago. ${ }^{1}$ As noted previously, this intriguing virus continues to mutate, evolves, and attempts to adapt to the host (i.e., us), and this may continue to be the state of play, at least for the immediate future. Several variants have evolved, more infectious and virulent, but not necessarily deadlier than the prototype strain sequenced in Wuhan, but notably also more seriously affecting the younger population. ${ }^{4-6}$ SARS-CoV-2 vaccines were developed and approved for use in a previously unachievable timeframe, with the first regulatory approvals seeing vaccine use begin at the end of 2020. At the time of writing, 20 vaccines have been authorized by at least one national regulatory authority for public use: two RNA vaccines (Pfizer-BioNTech and Moderna), nine conventional inactivated vaccines (BBIBP-CorV, Chinese Academy of Medical Sciences, CoronaVac, Covaxin, CoviVac, COVIran Barakat, Minhai-Kangtai, QazVac, and WIBP-CorV), five viral vector vaccines (Sputnik Light, Sputnik V, Oxford-AstraZeneca, Convidecia, and Janssen), and four protein subunit vaccines (Abdala, EpiVacCorona, MVC-COV1901, Soberana 02, and ZF2001). ${ }^{7}$ Heterologous vaccine administration (i.e., priming dose with one vaccine formulation, followed by a boost with another vaccine formulation) has also recently emerged as an intriguing opportunity for enhancing the generation of anti-SARS-CoV-2 neutralizing antibodies, as compared with homologous vaccination, especially when lower immunogenicity is expected (i.e., older age, immune compromise, cancer, impaired renal function, etc.). In addition, according to Wikipedia, a total of "330 vaccine candidates are in various stages of development, with 102 in clinical research, including 30 in Phase I trials, 30 in Phase I to II trials, 25 in Phase III trials, and eight in Phase IV development."7 It may be that even these may need continuing supplementation, given the rate of mutation and variant emergence. Another concern is that despite the ongoing COVID-19 vaccination campaigns, these drives
Address for correspondence Emmanuel J. Favaloro, PhD, FFSc (RCPA), Department of Haematology, Institute of Clinical Pathology and Medical Research (ICPMR), Westmead Hospital, Westmead 2145, Australia (e-mail: emmanuel. favaloro@health.nsw.gov.au).
Issue Theme Maintaining Hemostasis and Preventing Thrombosis in COVID-19-Part III; Guest Editors: Emmanuel J. Favaloro, PhD, FFSc (RCPA) and Giuseppi Lippi, $M D$ (c) 2022. Thieme. All rights reserved. Thieme Medical Publishers, Inc., 333 Seventh Avenue, 18th Floor, New York, NY 10001, USA
DOI https://doi.org/ 10.1055/s-0041-1736169. ISSN 0094-6176. 
have been variably successful, mainly due to issues around vaccine supply, access, equity and even vaccination hesitancy, in part driven by misinformation in social media.

Given the continued and vast explosion of information, it is impossible for anyone to keep up with the literature on COVID-19. Also, given the plethora of information, it becomes increasingly difficult to determine what novel information can, or should be added to the list. In the first STH issue on COVID-19, ${ }^{1}$ the editorial team of STH contributed enormously by generating a series of Commentaries, which have indeed proved very popular. ${ }^{2}$ One situation that was clear to us at that time was that given the great interest and initial thirst for knowledge, all journals became very interested in publishing on COVID-19 and, indeed, many papers were fast-tracked to publication, and some have since also been retracted. The STH board decided that although STH needed to publish on COVID-19, it did not want to publish just anything on COVID-19, and so a fairly resistant stance to acceptance of COVID-19 papers, just because they mentioned COVID-19, was undertaken. Clearly, STH did not want to publish misinformation, or even just add other "me too" papers (e.g., reviews similar to those that had already been published elsewhere). The second issue thus took some 6 months to accumulate enough material to collate into an issue, ${ }^{2}$ and this third issue has taken another 8 months. We continue to resist publishing on COVID-19 just for the sake of publishing on this compelling matter, since just adding more "noise" to the literature may hinder, rather than help the cause.

Given the emergence of vaccines against SARS-CoV-2, it is perhaps timely then to provide some information on one of the rare adverse effects of immunization, and namely COVID-19 vaccine-induced (immune) thrombosis with thrombocytopenia (VITT), also called thrombosis with thrombocytopenia syndrome (TTS). Written by the Guest Editors to this issue on COVID-19, this review is focused on perhaps the most severe presentation of VITT/TTS, namely cerebral venous thrombosis (CVT). ${ }^{8}$ As for many other infectious diseases, COVID-19 vaccination has now become crucial for limiting viral spread, especially for averting hospitalizations, need for intensive care, and fatal outcome. Nonetheless, as for other vaccines, COVID-19 vaccination is not completely free from side effects. Among the adverse events that have been reported after receiving COVID-19 vaccination, special emphasis has been given to an unexpected number of thrombocytopenic episodes, with or without thrombotic complications, especially in recipients of adenovirus-based COVID-19 vaccines. Along with a specific clinical presentation, encompassing "atypical" thrombosis (especially cerebral venous [sinus] thrombosis, CVT) prevalent in young female subjects, this new syndrome called VITT is characterized by, and thereby diagnosed for, the presence of three paradigmatic laboratory abnormalities, i.e., low platelet count $\left(<150 \times 10^{9} / \mathrm{L}\right)$, elevated plasma D-dimer levels (typically $>0.5 \mathrm{mg} / \mathrm{L}$ ), accompanied by a positive test for anti-PF4 (platelet factor 4) antibodies assayed with ELISA techniques. ${ }^{9}$ Timely identification of these important abnormalities by both clinicians and laboratory professionals seems therefore vital for early diagnosis and management of VITT, since this condition may have fatal outcome in up to half (or even more) of affected patients, depending on clinical presentation. This narrative review aims to review the epidemiology, pathogenesis, clinical and laboratory characteristics of VITT and other COVID-19 vaccine-associated thrombocytopenias.

Next in this issue, Jecko Thachil provides a separate Commentary on VITT, discussing the "shades of grey in thrombus formation." 10 Historically, two types of clots have been described in the medical literature-the so called "white clot," where the platelets appear to dominate, and the so called "red clot," where fibrin network with trapped red blood cells represent the main elements of the thrombi. A vessel-based distinction has also been identified, where platelet-rich clots occur in arterial thrombosis, while activation of coagulation factors is mostly proposed to cause venous thrombosis. Accordingly, antiplatelet drugs are the treatment of choice where arterial thrombosis occurs, whereas anticoagulants are the typical drug therapy in venous thrombosis. With both platelets and the clotting system involved in VITT, Dr. Thachil poses several questions, including: can VITT provide clues to the observed shades of gray (rather than a clear red and white distinction) often seen in thrombus formation? And should we consider more than just antiplatelets or anticoagulants in treating or preventing extension of thrombus formation in the future?

Naturally, despite the devastation that VITT may cause, COVID-19 is by far a worse condition. As a pandemic, it is inevitable that all of us will be exposed to SARS-CoV-2 in time, with a risk of death of around $5 \%$ overall, increasing for those with comorbidities, advanced age, and extremes of body weight. In contrast, the relative risk of dying from VITT is around one in a million vaccinations. Accordingly, the majority of this issue is focused on COVID-19.

First, Bahraini and Dorgalaleh reviewed the prothrombotic changes caused by COVID-19, and specifically the impact of SARS-CoV-2 infection on blood coagulation and fibrinolytic pathways. ${ }^{11}$ The cardinal pathology of COVID-19 is a primary infection of pulmonary tract cells by SARS-CoV-2, provoking a local inflammatory response, often accompanied by the socalled "cytokine storm" and acute respiratory distress syndrome (ARDS), especially in patients with severe/critical illness. Systemic propagation of disease may associate with thrombotic events, including deep vein thrombosis, pulmonary embolism, and thrombotic microangiopathy, which are important causes of morbidity and mortality in patients with COVID-19. This narrative review thus describes current knowledge of the pathophysiological mechanisms of COVID-19associated coagulopathy, with focus on prothrombotic changes in hemostatic mediators, including plasma levels of clotting factors, natural anticoagulants, components of fibrinolytic system and platelets. It also highlights the central role of endothelial cells in COVID-19-associated coagulopathy and discusses potential therapeutic strategies for managing thrombotic complications. Awareness by medical experts of contributors to the pathogenesis of thrombotic events in COVID-19 is imperative to develop therapeutics not limited to regular anticoagulants. Instituting cooperation among 
medical personnel and researchers may lessen the viral impact now, and in the event of recurrence.

Next, Hvas and colleagues describe the changes in dynamic hemostasis and fibrinolysis assays arising in intensive care COVID-19 patients, and their association with thrombosis and bleeding in a hybrid article, as provided by a systematic review, but also including some original data. ${ }^{12}$ Patients admitted to the intensive care unit (ICU) with COVID-19 have a high risk of thrombosis, though the precise mechanisms behind this remain unclarified. The authors undertook a systematic literature search in PubMed and EMBASE, and identified 18 prospective studies applying dynamic coagulation assays in ICU COVID-19 patients. Overall, these studies revealed normal or slightly reduced primary hemostasis, prolonged clot initiation but increased clot firmness. Thrombin generation assay parameters generally were equivalent to the control groups or within reference range. Fibrinolysis assays showed increased clot resistance. Only six studies related their findings to clinical outcomes. The authors also prospectively included 51 COVID-19 patients admitted to the ICU. Blood samples were examined on day 1,3 to 4 , and 7 to 8 with platelet function tests, rotational thromboelastometry (ROTEM), in vivo and ex vivo thrombin generation, and clot lysis assay. Data on thrombosis, bleeding, and mortality was recorded during 30 days. Primary hemostasis was comparable to healthy controls, but COVID-19 patients had longer ROTEM-clotting times and higher maximum clot firmness than healthy controls. Ex vivo thrombin generation was similar to that of healthy controls, while in vivo thrombin generation markers, thrombin-antithrombin (TAT) complex and prothrombin fragment $1+2(\mathrm{~F} 1+2)$, were higher in ICU COVID-19 patients than in healthy controls. Impaired fibrinolysis was present at all time points. TAT complex and $\mathrm{F} 1+2$ levels were significantly higher in patients developing thrombosis $(n=16)$ than in those without. The authors conclude that only a few previous studies employed dynamic hemostasis assays in COVID-19 ICU-patients, and these generally failed to reveal a clear association with the risk of developing thrombosis. In ICU COVID-19 patients, they also confirmed normal platelet aggregation, while in vivo thrombin generation was increased, and fibrinolysis decreased. The authors hypothesize that thrombosis may be driven by increased thrombin formation in vivo.

Next, the writing team of Favaloro, Henry, and Lippi provide two separate reviews related to COVID-19, and the presence of lupus anticoagulant (LA) and antiphospholipid antibodies (aPLs) in COVID-19 patients. ${ }^{13,14}$ Indeed, LA identifies a form of aPL that causes prolongation of clotting tests in a phospholipid concentration-dependent manner and represents one of the laboratory criteria identified in patients with antiphospholipid antibody syndrome (APS). The presence of LA in patients with APS represents a significant risk factor for both thrombosis and pregnancy morbidity. There have also been several reports of similarities between some of the pathophysiological features of COVID-19 and APS, in particular the most severe form, catastrophic APS (CAPS). There have also now been many reports identifying various aPLs, including LA, in COVID-19 patients. Accordingly, a very pertinent question arises: "is LA a feature of COVID-19 pathology?" In the first review, ${ }^{13}$ the authors critically appraise the literature to help answer this question. They conclude that LA positivity is a feature of COVID-19, at least in some patients, and potentially those who are the sickest or have the most severe infection. However, many publications have failed to appropriately consider the many confounders to LA identification, being assessed using clot-based assays such as the dilute Russell Viper Venom Time, the activated partial thromboplastin time (aPTT), and the silica clotting time. First, most patients hospitalized with COVID-19 are placed on anticoagulant therapy, and those with prior histories of thrombosis would possibly present to hospital already on anticoagulant therapy. All anticoagulants, including vitamin K antagonists, heparin (both unfractionated heparin and low molecular weight heparin), and direct oral anticoagulants affect these clotbased assays. Second, C-reactive protein (CRP) is highly elevated in COVID-19 patients, and also associated with severity. CRP can also lead to false positive LA, particularly using the aPTT assay. Third, persistence of aPL positivity (including LA) is required to identify APS. Fourth, those at greatest risk of thrombosis due to aPL are those with highest titers or multiple positivity. Most publications either did not identify anticoagulation and/or CRP in their COVID-19 cohorts, nor did they seem to account for these as possible confounders for LA detection. Most publications did not assess for aPL persistence, and where persistence was checked, LA appeared to represent transient aPL. Finally, high titer aPL or multiple aPL positivity was in the minority of COVID-19 presentations. Thus, at least some of the reported LA associated with COVID-19 are likely to be false positives, and the relationship between the detected aPL/LA and COVID-19 associated coagulopathy remains to be resolved using larger and better studies. The second review ${ }^{14}$ is focused primarily on solid-phase detected aPL. Although LA can be identified as a form of aPL, solid-phase detected aPLs are not affected by anticoagulant therapy or CRP, and thus these confounders will not cause false test results in such assays. These aPLs comprise "criteria-aPL," such as anticardiolipin antibodies and anti- $\beta 2$-Glycoprotein I antibodies (ab2GPI) of the immunoglobulin (Ig) G and M classes, and "non-criteria antibodies," which include, for example, antiphosphatidylserine antibodies (aPS), antiprothrombin antibodies (aPT), and antiphosphatidylserine/prothrombin complex antibodies (aPS/PT). In this review, the authors critically appraise the literature on aPL and COVID-19. This can be considered a companion piece to the earlier review focused on LA. ${ }^{13}$ Nevertheless, the authors conclude similarly that aPL positivity may be a feature of COVID-19, at least in some patients, but in general, identified "solid phase" aPLs are of low titer and not able to be well-linked to the thrombotic aspects of COVID-19. Also, most publications again did not assess for aPL persistence, and where persistence was checked, the findings appeared to represent transient form of aPL. Importantly, high titer aPL or multiple aPL positivity (including double, triple) was in the minority of COVID-19 presentations, and thus discounts any 
widespread presence of APS, including the most severe form of CAPS, in COVID-19 patients.

Next is a review by Poredos and Poredos, who provide an update on the involvement of inflammation in venous thromboembolic disease, with special focus on COVID-19. ${ }^{15}$ The authors begin by reminding readers that the inflammatory process is strongly involved in the pathophysiology of venous thromboembolism (VTE) and has a significant role in disease prediction. Inflammation most probably represents a common denominator through which classical and non-classical risk factors stimulate thrombotic process. Inflammation of venous wall promotes the release of tissue factor, inhibits the release of anticoagulant factors, and hampers endogenous fibrinolysis. Systemic inflammatory response also inhibits restoration of blood flow in occluded vessels. Recent studies further indicate that increased inflammatory response ("cytokine storm") is related to both the prothrombotic state and thromboembolic events in patients with COVID-19. The growing evidence of involvement of inflammation in the pathogenesis of VTE indicates the potential role of anti-inflammatory treatment and prevention of VTE. While aspirin was shown to be effective in preventing recurrent venous thrombosis after treatment with anticoagulant drugs, some other anti-inflammatory drugs like nonsteroidal anti-inflammatory agents may have prothrombotic effect, thus potentially increasing the risk of VTE. Recently, new specific anti-inflammatory drug inhibitors of inflammatory markers that have been shown to be involved in the pathogenesis of VTE are being investigated. As thrombogenesis is based on activation of coagulation provoked by inflammation, then prevention and treatment of VTE should include both anticoagulant and anti-inflammatory agents. Combined treatment is related to increased risk of bleeding complications, therefore sub-therapeutic doses of both drugs should be used to improve the efficacy of management of VTE, in COVID-19 and otherwise, without increasing risk of bleeding.

The last full-length paper in this STH issue is another hybrid paper by Abate and collegues. ${ }^{16}$ The authors begin by reminding readers that coagulation abnormalities, thrombosis, and endothelial dysfunction have been well described in COVID-19 patients. However, spontaneous muscle hematoma $(\mathrm{SMH})$ represents a rare complication of COVID-19. The aims of this paper are to: (1) report on a systematic review of the literature to better define the clinical SMH characteristics, and (2) describe prevalence and clinical characteristics of SMH in COVID-19 patients referring to several hospitals. In their retrospective analysis, the authors describe 10 cases of COVID-19 patients with SMH not previously reported in literature, with a prevalence of $2.1 \%$. The literature review, inclusive of their case series, describes a total of 50 SMHs in COVID-19 patients (57.4\% males; mean age $68.8 \pm 10.0$ years). The SMH sites were ileo-psoas, vastus intermedius, gluteus, sternocleidomastoid, and pectoralis major muscles. Males developed SMH earlier than females (9.5 \pm 7.8 vs. $17.1 \pm 9.7$ days). Ileo-psoas hematoma was more frequent in males (69.2 vs. $30.8 \%$ ), while pectoralis major hematoma occurred only in females. The in-hospital mortality rate of
SMH in COVID-19 patients was $32.4 \%$. The authors concluded that SMH is a rare but severe complication in COVID-19 hospitalized patients, associated with high mortality. A gender difference also seems to be present in the clinical presentation of the disorder.

The remainder of this issue contains several Letters to the Editor. ${ }^{17-22}$ Such "correspondence" permits publication of various "smaller" COVID-19 vignettes. First comes a paper related to VITT. Limami et al provide data to show that despite causing VITT in a small proportion of vaccinated patients, the Oxford-AstraZeneca ChAdOx1 COVID-19 vaccine does not significantly alter platelet aggregation. Briefly, the authors assessed subjects vaccinated with a single dose $(n=35)$ or with two doses $(n=42)$ of vaccine, as well as patients with severe COVID-19 $(n=10)$. Using washed platelets and plateletrich plasma from these individuals, the authors showed that platelets from COVID-19 patients were hyperreactive, in confirmation of previous studies. However, platelets from vaccinated subjects were not found to be hyperreactive. Next, Fan and Cheung remind us that not only venous thrombosis, but also arterial thrombosis can be a feature of COVID-19. ${ }^{18}$ Moreover, while an increased incidence of thromboembolic events and hypercoagulability during acute SARS-CoV-2 infection have been well described, arterial thrombosis postCOVID-19, although uncommon, has yet to be recognized as part of the late sequelae of COVID-19 (i.e., the so-called "longCOVID" syndrome). This is despite the emerging reports of post-COVID-19 arterial thrombosis, particularly in patients without cardiovascular risk factors. Fazio and colleagues then provide data on the longer-term pattern of $\mathrm{D}$-dimer in patients who have recovered from mild to moderate COVID-19. ${ }^{19}$ The authors report evidence of persisting high D-dimer levels at 15 days after complete clinical and laboratory remission in $20 \%$ of 45 assessed patients. Next, Weatherill and colleagues report on the impact of thrombosis and bleeding in patients with severe COVID-19 versus other viral pneumonias in the context of extracorporeal membrane oxygenation (ECMO), being a treatment potentially life-saving in people with severe COVID-19. ${ }^{20}$ The report describes a single-center retrospective observational study in a UK ECMO center to assess whether thrombotic or bleeding phenotypes differed in patients with severe COVID-19 pneumonia treated with veno-venous (VV)ECMO compared with those who received VV-ECMO for other viral pneumonias. Mortality rate up to 120 days after VVECMO initiation was also assessed, as well as the factors directly contributing to death. To summarize findings, the authors found that thrombosis and major bleeding were frequent complications in both COVID-19 and nonCOVID-19 patients supported with VV-ECMO. Their principal finding was that, although thrombosis is a dominant feature of COVID-19 infection, bleeding was the major cause of death in patients supported with VV-ECMO, accounting for five out of nine deaths in COVID-19, compared with none in non-COVID19 patients. Finally, for this issue of STH, Chiurazzi and colleagues describe a rare case of an acquired factor $V$ inhibitor after COVID-19. ${ }^{21}$ Nevertheless, a causal relationship in their case (and of course in other published cases) remains unproven, and coincident findings of acquired inhibitors and COVID- 
19 are possible, as are coincident findings of acquired inhibitors and COVID-19 vaccination. ${ }^{22}$

We thank all the authors to this third issue of "maintaining hemostasis and preventing thrombosis in COVID19 " for their contributions, and we hope that the readership of STH will enjoy this third installment in this now continuing series. We ultimately hope that the global vaccination program acts to dampen the pandemic scourge that is COVID-19, which provides a risk of morbidity and mortality many folds higher than the overall rare risk of VITT/TTS, even in its most severe presentation as $\mathrm{CVT}^{8}$ Given the severe coagulopathy associated with COVID-19, ${ }^{23}$ and the assured continuance of this pandemic for the foreseeable future, we expect to be back for a fourth instalment of "Maintaining Hemostasis and Preventing Thrombosis in Coronavirus Disease 2019 (COVID-19)" in the near future.

\section{Conflict of Interest}

None declared.

\section{References}

1 Favaloro EJ, Lippi G. Maintaining hemostasis and preventing thrombosis in coronavirus disease 2019 (COVID-19)-part I. Semin Thromb Hemost 2020;46(07):757-762

2 Favaloro EJ, Lippi G. Maintaining hemostasis and preventing thrombosis in coronavirus disease 2019 (COVID-19)-part II. Semin Thromb Hemost 2021;47(04):333-337

3 COVID-19 Dashboard by the Center for Systems Science and Engineering (CSSE) at Johns Hopkins University (JHU). Accessed August 11, 2021 at: https://www.arcgis.com/apps/opsdashboard/ index.html\#/bda7594740fd40299423467b48e9ecf6

4 Tregoning JS, Flight KE, Higham SL, Wang Z, Pierce BF. Progress of the COVID-19 vaccine effort: viruses, vaccines and variants versus efficacy, effectiveness and escape. Nat Rev Immunol 2021;21(10): 626-636

5 Sharma K, Koirala A, Nicolopoulos K, Chiu C, Wood N, Britton PN. Vaccines for COVID-19: where do we stand in 2021? Paediatr Respir Rev 2021;S1526-0542(21)00065-8

6 Raman R, Patel KJ, Ranjan K. COVID-19: unmasking emerging SARS-CoV-2 variants, vaccines and therapeutic strategies. Biomolecules 2021;11(07):993

7 COVID-19 vaccines. Accessed August 11, 2021 at: https://en. wikipedia.org/wiki/COVID-19_vaccine

8 Lippi G, Favaloro EJ. Cerebral venous thrombosis developing after COVID-19 vaccination: VITT, VAT, TTS and more. Semin Thromb Hemost 2022;48(01):8-14
9 Favaloro EJ. Laboratory testing for suspected COVID-19 vaccineinduced (immune) thrombotic thrombocytopenia. Int J Lab Hematol 2021;43(04):559-570

10 Thachil J. COVID-19 vaccine induced immune thrombosis with thrombocytopenia (VITT) and the shades of grey in thrombus formation. Semin Thromb Hemost 2022;48(01):15-18

11 Bahraini M, Dorgalaleh A. The impact of SARS-CoV-2 infection on blood coagulation and fibrinolytic pathways: a review of prothrombotic changes caused by COVID-19. Semin Thromb Hemost 2022;48(01):19-30

12 Hvas CL, Larsen JB, Adelborg K, Christensen S, Hvas A-M. Dynamic hemostasis and fibrinolysis assays in intensive care COVID-19 patients and association with thrombosis and bleeding-a systematic review and a cohort study. Semin Thromb Hemost 2022; 48(01):31-54

13 Favaloro EJ, Henry BM, Lippi L. Is lupus anticoagulant a significant feature of COVID-19? A critical appraisal of the literature. Semin Thromb Hemost 2022;48(01):55-71

14 Favaloro EJ, Henry BM, Lippi L. COVID-19 and antiphospholipid antibodies: time for a reality check? Semin Thromb Hemost 2022; 48(01):72-92

15 Poredos P, Poredos P. Involvement of inflammation in venous thromboembolic disease: an update in the age of COVID-19. Semin Thromb Hemost 2022;48(01):93-99

16 Abate V, Casoria A, Rendina D, et al. Spontaneous muscle hematoma in patients with COVID-19: a systematic literature review with description of an additional case series. Semin Thromb Hemost 2022;48(01):100-108

17 Limami Y, Khalki L, Zaid N, et al. Oxford-AstraZeneca ChAdOx1 COVID-19 vaccine does not alter platelet aggregation. Semin Thromb Hemost 2022;48(01):109-111

18 Fan BE, Cheung C. Post COVID-19 arterial thromboembolism: a clear and present danger. Semin Thromb Hemost 2022;48(01):112-114

19 Fazio S, Tufano A, de Simone G. Sustained high D-dimer in outpatients who have recovered from mild to moderate coronavirus disease 2019 (COVID-19). Semin Thromb Hemost 2022;48(01):115-117

20 Weatherill A, Laffan M, Gasper G, et al. Impact of thrombosis and bleeding in patients with severe COVID-19 vs other viral pneumonias in the context of extracorporeal membrane oxygenation. Semin Thromb Hemost 2022;48(01):118-123

21 Chiurazzi F, Tufano A, Esposito M, D'Agostino F, Casoria A, Capasso F, Di Minno G. Acquired factor $\mathrm{V}$ inhibitor after coronavirus disease 2019 (COVID-19). Semin Thromb Hemost 2022;48(01):124-126

22 Cittone MG, Battegay R, Condoluci A, et al. The statistical risk of diagnosing coincidental acquired hemophilia A following antiSARS-CoV-2 vaccination. J Thromb Haemost 2021;19(09): 2360-2362

23 Lippi G, Sanchis-Gomar F, Favaloro EJ, Lavie CJ, Henry BM. Coronavirus disease 2019-associated coagulopathy. Mayo Clin Proc 2021;96(01):203-217 